\title{
The priming induction regimen of HAG as a low dose chemotherapy strategy in AML clonal evolution
}

\author{
CHEN AiLi ${ }^{1}$, YANG Jing Yi $^{1}$, HU ShaoYan ${ }^{2 *} \&$ WANG Qian-Fei ${ }^{1 *}$ \\ ${ }^{1}$ Key Laboratory of Genomic and Precision Medicine, Beijing Institute of Genomics, Chinese Academy of Sciences, Beijing 100101, China; \\ ${ }^{2}$ The Children's Hospital of Soochow University, Suzhou 215003, China
}

Received August 22, 2015; accepted September 16, 2015; published online November 19, 2015

Citation: $\quad$ Chen AL, Yang JY, Hu SY, Wang QF. The priming induction regimen of HAG as a low dose chemotherapy strategy in AML clonal evolution. Sci China Life Sci, 2015, 58: 1302-1305, doi: 10.1007/s11427-015-4974-5

Chemotherapy employs chemical substances to interfere with the growth of cancer cells, and is a major treatment strategy in human cancer including acute myeloid leukemia (AML). Although they often effectively kill fast-dividing tumor cells, chemotherapeutic drugs also profoundly affect mutation spectrum of the tumor genome. Each chemotherapeutic drug has a working concentration within an appropriate range. Concentrations beyond this range will increase drug toxicity without adding clinical benefit, while concentrations below the range will result in an insufficient drug effect. The conventional strategy in oncology is to give a patient drugs at the maximum tolerated dose (MTD) in order to kill as many tumor cells as possible. However, this inevitably causes a high rate of toxicity to normal tissue, as well as resulting in new mutations and genome instability which can lead to drug resistance and relapse. The priming induction regimen of HAG (low dose of cytarabine (Ara-C) and Homoharringtonine (HHT) with granulocyte colony-stimulating factor (G-CSF) priming), has proven to be effective in subgroup of AML patients in small scale clinical studies. It is of great medical significance to explore whether low dose chemotherapy can reduce the side effect of chemotherapeutic drugs while maintaining the therapeutic benefits, and whether such alternative therapeutic strategy is advantageous compared to conventional intensive treatment regarding the development of drug resistance by affecting leukemic genome mutation load and clonal evolu-

*Corresponding author (email: wangqf@big.ac.cn; hsy139@126.com) tion in subgroup of AMLs.

\section{Clonal evolution and drug resistance after conventional chemotherapy in AML}

Despite the improvement in conventional intensive chemotherapy and stem cell transplantation, the overall 5-year survival of AML patients is only $40 \%-45 \%$ in young patients and less than $10 \%$ in the elderly patients. The majority of patients relapse with resistance to chemotherapy, and the cancer rapidly progresses. These tumors have undergone clonal evolution at the cytogenetic level, which is caused by a potent selection pressure from the chemotherapy [1].

Although intensive chemotherapy is designed to eradicate all tumor clones in a patient, recent studies show that it not only fails to kill the founding clone but also increases the risk of relapse by directly inducing new mutations that contribute to cancer evolution. By sequencing the whole genomes of 8 AML patients, Ding et al. [2] reveal two major clonal evolution patterns at relapse after chemotherapy: either the founding clone in the primary tumor gained mutations and evolved into a relapse clone or a subclone of the founding clone survived the initial therapy, gained additional mutations and expanded at relapse. Moreover, it has been proposed that relapse-specific drug resistance mutations are likely to have been present in small populations of the founding clones, and intensive chemotherapy, which kills the maximum numbers of those founding clones, removes 
the inhibitory effect on the small clones and eventually promotes more rapid growth of the resistant populations [3]. With such a potential threat of conventional chemotherapies, there is a critical need to adopt a new therapeutic concept of chemotherapy in cancer treatment.

\section{Priming induction regimen of CAG or HAG chemotherapy in treating AML}

Conventional chemotherapy can achieve complete remission (CR) in $60 \%-80 \%$ of de novo AML patients. However, it also has limited efficacy in the remaining 20\%-40\% of de novo AML patients, especially in high-risk AML patients including elderly and relapsed/refractory (R/R) patients. As a result of drug resistance, poor performance status (PS), multiple organ dysfunction and high treatment-related toxicities, conventional chemotherapy is associated with a high early death (ED) rate. In an attempt to achieve reduced toxicity, better tolerance and improved life quality, a priming induction regimen, CAG (lower dose of Ara-C (1/10 dosage of conventional therapy) and aclarubicin (ACR) with G-CSF priming), was first proposed in 1995 by a Japanese group [4]. ACR is an anthracycline drug with an adverse effect of cardiotoxicity, and Ara-C is an antimetabolism agent targeting pyrimidine synthesis. As a sensitizer of $S$ phase-specific cytotoxicity drugs, G-CSF can stimulate resting G0 phase cells into the G1 phase and mobilize the bone marrow leukemia cells to the peripheral blood. Following the priming of G-CSF, a combination of ACR with Ara-C is presumed to eliminate the leukemia cells with a higher efficiency [5]. In China, HHT, an alkaloid isolated from the Cephalotaxus plant, was used to replace ACR because HHT is less expensive and has a low risk of cardiotoxicity. Therefore, the CAG regimen is referred to as HAG when HTT is used in place of ACR. Although an increasing number of studies involve $\mathrm{CAG}$ and HAG regi- mens to treat AML, MDS and even ALL, priming induction regimens are still mainly used in elderly, $R / R$ and secondary AML patients as a second line treatment exclusively in China and Japan.

To systematically evaluate the efficacy of the CAG/HAG regimen in AML patients, we searched several databases, including PubMed and Web of Science, as well as the American Society of Hematology (ASH) and American Society of Clinical Oncology (ASCO) annual meeting abstracts between 2009 and 2015 for clinical studies. Preclinical trials, case reports and reviews were not included. Ten studies with a total of 416 patients were found (Table 1). Seven studies involving 250 AML patients used CAG and achieved a highly variable CR rates $(21.2 \%$ to $63.5 \%) .166$ AML patients from four of the studies were under HAG treatment, and the CR rates ranged from $46.7 \%$ to $61.5 \%$. The overall CR rates calculated with a random effect model in the AML patients were $47.2 \%$ (95\% confidence interval (CI), 35.2\%-59.2\%) and 57.0\% (95\%CI, 50.3\%-63.7\%) for CAG or HAG, respectively. CAG, HAG and conventional standard regimens had similar $\mathrm{CR}$ rates in these studies (Fisher test $P>0.05$ ). Additionally, CAG and HAG achieved similar CR rates in either 136 de novo or $280 \mathrm{R} / \mathrm{R}$ AMLs $(49.0 \%$ versus $46.5 \%$, 60.7\% versus $56.0 \%$, Fisher test $P>0.05$ ). More recently, Li et al. [6] reported in Oncotarget that $\mathrm{CAG}$ in combination with decitabine achieved a $\mathrm{CR}$ rate of $82.4 \%$ in de novo elderly AML patients.

The toxicity of the CAG/HAG regimen in all of the reports was generally mild. Early death was reported in 13 out of 388 patients $(3.4 \%)$, and no patient suffered from cardiotoxicity. Most of the deaths were caused by severe infection, respiratory failure and cerebral vascular accident, which were significantly lower than the outcomes observed when conventional induction therapy was used. These data indicated that the CAG/HAG regimen is safe and effective as a promising induction therapy in $\mathrm{AML}$, for both de novo and R/R patients. However, the overall survival (OS), pro-

Table 1 Efficacy, cardiotoxicity and early death rate of CAG and HAG in AML ${ }^{\text {a) }}$

\begin{tabular}{|c|c|c|c|c|c|c|c|c|c|c|c|}
\hline & Author & Year & Patients No. & $\begin{array}{c}\text { Median } \\
\text { age }(y)(\text { range })\end{array}$ & ACR/HHT dosage & Subtype & CR No. & CR \% & OS (m)(range) & CT No. & ED No. \\
\hline \multirow{7}{*}{$\begin{array}{l}\mathrm{CAG} \\
\text { regimen }\end{array}$} & Su et al. & 2009 & 33 & $60(28-77)$ & $10 \mathrm{mg} \times 8 \mathrm{~d}$ & $\mathrm{RR}$ & 14 & 42.4 & NA & 0 & 0 \\
\hline & Wei et al. & 2012 & 23 & $41(14-60)$ & $8-10 \mathrm{mg} \mathrm{m}^{-2} \times 8 \mathrm{~d}$ & $\mathrm{RR}$ & 13 & 56.5 & NA & 0 & 0 \\
\hline & Liu et al. & 2014 & 37 & $46(15-76)$ & $5-7 \mathrm{mg} \mathrm{m}^{-2} \times 14 \mathrm{~d}$ & $\mathrm{RR}$ & 23 & 62.2 & $6(2-36)$ & 0 & 0 \\
\hline & Wang et al. & 2014 & 33 & $2(15-66)$ & $20 \mathrm{mg} \times 4 \mathrm{~d}$ & $\mathrm{RR}$ & 7 & 21.2 & NA & 0 & 0 \\
\hline & Zheng et al. & 2012 & 28 & $68(60-89)$ & $20 \mathrm{mg} \times 3$ or $4 \mathrm{~d}$ & de novo & 12 & 42.9 & $10.5(0.6-64.3)$ & 0 & NA \\
\hline & Zhu et al. & 2013 & 44 & $45(16-62)$ & $10 \mathrm{mg} \times 8 \mathrm{~d}$ & RR & 28 & 63.5 & NA & 0 & 0 \\
\hline & Jin et al. & 2015 & 52 & $68(60-80)$ & $6-7 \mathrm{mg} \mathrm{m}^{-2} \times 14 \mathrm{~d}$ & de novo & 29 & 55.5 & $12.1(0.65-11.7)$ & 0 & 2 \\
\hline \multirow{4}{*}{$\begin{array}{l}\text { HAG } \\
\text { regimen }\end{array}$} & Su et al. & 2009 & 30 & $68(17-88)$ & $1 \mathrm{mg} \times 14 \mathrm{~d}$ & $\mathrm{RR}$ & 14 & 46.7 & NA & 0 & 0 \\
\hline & Wu et al. & 2011 & 13 & 71(60-88) & $1 \mathrm{mg} \times 14 \mathrm{~d}$ & $\mathrm{RR}$ & 8 & 61.5 & NA & 0 & 0 \\
\hline & Gu et al. & 2011 & 67 & $50(15-77)$ & $1.5 \mathrm{mg} \mathrm{m}^{-2} \times 14 \mathrm{~d}$ & RR & 35 & 52.2 & $12.5(0.5-37)$ & 0 & 7 \\
\hline & Chen et al. & 2015 & 56 & $72(60-80)$ & $1.5 \mathrm{mg} \mathrm{m}^{-2} \times 14 \mathrm{~d}$ & de novo & 34 & 60.7 & $12(8.6-15.4)$ & 0 & 4 \\
\hline
\end{tabular}

a) AML: acute myeloid leukemia; CR: complete remission; OS: overall survival; CT: cardiotoxicity; ED: early death; NA: not available; CAG: Ara-C, ACR and G-CSF; HAG: HHT, Ara-C and G-CSF. 
gression free survival (PFS) and relapse rate were rarely reported in these studies. Further large-scale prospective, randomized controlled investigations should be performed to systematically evaluate the long-term efficacy of the CAG/HAG regimen.

\section{Priming induction regimen of chemotherapy and clonal evolution in AML}

Over the last six years, several groups applied mathematical models to define the patterns of tumor clonal evolution under the treatment of different chemotherapeutic drug dosage. They proposed that, there are pre-existing small populations of chemotherapy-resistant subclones in cancer patients and that the presence of the founding clones can suppress the expansion of the resistant clones. By killing most of the cells in a founding clone, conventional chemotherapy also removes this inhibitory effect and eventually promotes the rapid growth of the resistant populations. Alternatively, if a therapy allows the founding clone to survive at a tolerated tumor load, the founding clone will, in turn, suppress the growth of the resistant population $[3,7,8]$. Their models provide a novel therapeutic concept and support the validity of low dose chemotherapy.

By imparting a reduced selection pressure on the leukemic genome and clonal structure, the priming induction regimen of HAG may give rise to a more beneficial clinical outcome in the long run. There is increasing evidence to show that a low(er) dose instead of an MTD therapy can maintain a minimum tumor burden, which in turn, may restrain the proliferation of the most resistant clones. A recent study indicated that the priming induction regimen might also help to prevent the quiescent cancer stem cells (CSC) to become activated and cause tumor progression and post-treatment cancer relapse [9]. Moreover, low dose chemotherapy is presumed to have less impairment on the bone marrow microenvironment and immune system. Together, these beneficial effects may lead to a better recovery of hematopoiesis after chemotherapy, as well as retain a sensitive response to the same regimen or similar chemotherapy upon relapse (Figure 1).

\section{Summary and perspectives}

Collectively, multiple small-scale clinical studies support the priming induction regimen of low doses administration as a promising alternative to conventional intensive strategy in AML induction therapy for high risk patients. The short-term efficacy, reduced side effect and lower costs of the priming induction regimen are evident, large-scale investigations are still needed to evaluate OS, PFS and relapse rate. Although it is not a focus of this review, alterna-

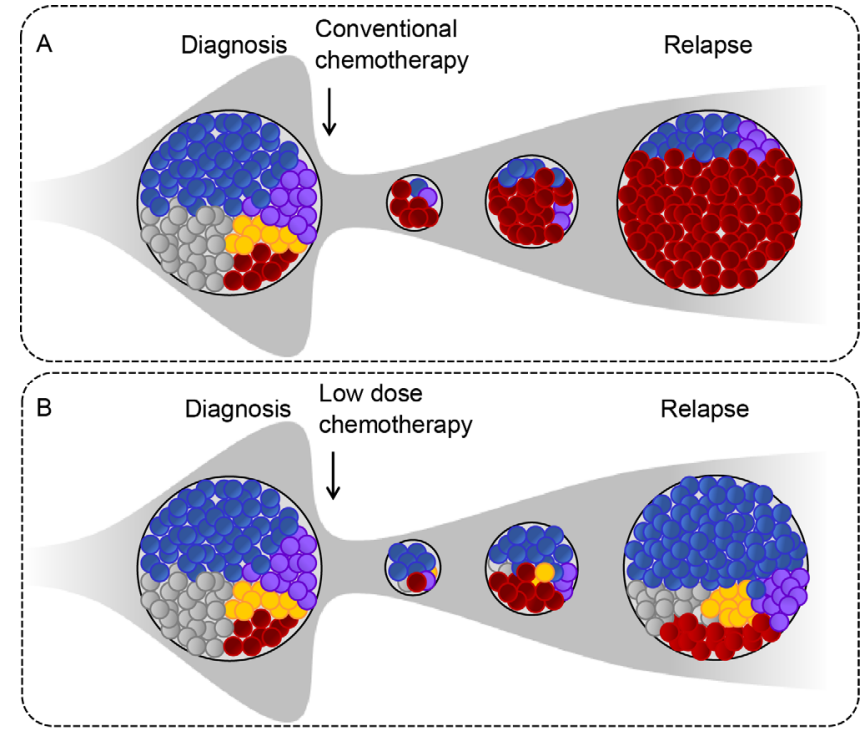

Figure 1 The distinct clonal evolution patterns under the selection of conventional or low-dose chemotherapy. A, High dose chemotherapeutic drugs eradicate most cells from the founding clone, and leave the pre-existing small populations of drug-resistant subclones to expand upon relapse. B, A proposed model: low drug dosage kills tumor cells without impacting the clonal structure, relapsed tumors are still sensitive to the same or similar chemotherapy regimens.

tive priming agents are also used in the clinic. For example, priming with dichloroacetate (DCA) significantly potentiated the cytotoxicity of arsenic trioxide (ATO) in AML cells in a synergistic manner. The combination decreased the mitochondrial membrane potential as well as expression of Mcl-1 and GPx in primary AML cells more than either drug alone [10]. Intriguing, mathematical modeling and recent genomic studies suggest a potential inhibitory effect of low dose chemotherapy on the evolution of drug-resistant clones. Future studies are warranted to determine if the distinct evolutionary course exerted by low dose chemotherapy may lead to improved clinical outcomes by reducing relapse and drug resistance, at least in subgroup of AML patients. Therefore, the decision can be made to choose "the right dose" in precision medicine.

The author(s) declare that they have no conflict of interest.

This work was supported by China Ministry of Science and Technology grants(2014CB542001), the Key Research Program of the Chinese Academy of Sciences (Precious Medicine Research in Chinese Population), the National Natural Science Foundation of China (91331111, 81425003).

1 Burrell RA, Swanton C. Tumour heterogeneity and the evolution of polyclonal drug resistance. Mol Oncol, 2014, 8: 1095-1111

2 Ding L, Ley TJ, Larson DE, Miller CA, Koboldt DC, Welch JS, Ritchey JK, Young MA, Lamprecht T, McLellan MD, McMichael JF, Wallis JW, Lu C, Shen D, Harris CC, Dooling DJ, Fulton RS, Fulton LL, Chen K, Schmidt H, Kalicki-Veizer J, Magrini VJ, Cook L, McGrath SD, Vickery TL, Wendl MC, Heath S, Watson MA, Link 
DC, Tomasson MH, Shannon WD, Payton JE, Kulkarni S, Westervelt P, Walter MJ, Graubert TA, Mardis ER, Wilson RK, DiPersio JF. Clonal evolution in relapsed acute myeloid leukaemia revealed by whole-genome sequencing. Nature, 2012, 481: 506-510

3 Diaz LA Jr, Williams RT, Wu J, Kinde I, Hecht JR, Berlin J, Allen B, Bozic I, Reiter JG, Nowak MA, Kinzler KW, Oliner KS, Vogelstein B. The molecular evolution of acquired resistance to targeted EGFR blockade in colorectal cancers. Nature, 2012, 486: 537-540

4 Yamada K, Furusawa S, Saito K, Waga K, Koike T, Arimura H, Aoyagi A, Yamato H, Sakuma H, Tsunogake S. Concurrent use of granulocyte colony-stimulating factor with low-dose cytosine arabinoside and aclarubicin for previously treated acute myelogenous leukemia: a pilot study. Leukemia, 1995, 9: 10-14

5 Saito K, Nakamura Y, Aoyagi M, Waga K, Yamamoto K, Aoyagi A, Inoue F, Nakamura Y, Arai Y, Tadokoro J, Handa T, Tsurumi S, Arai H, Kawagoe Y, Gunnji H, Kitsukawa Y, Takahashi W, Furusawa S. Low-dose cytarabine and aclarubicin in combination with granulocyte colony-stimulating factor (CAG regimen) for previously treated patients with relapsed or primary resistant acute myelogenous leukemia (AML) and previously untreated elderly patients with AML, secondary AML, and refractory anemia with excess blasts in transformation. Int J Hematol, 2000, 71: 238-244

6 Li J, Chen Y, Zhu Y, Zhou J, Xu Y, Li Y, Yu K, Pan L, Wang J, Ding J, Gu J, Zhou S, Shi J, Hong M, Xu J, Pan L, Duan L, Zhang R, Zhang S, Zhu H, Lu H, Liu P, Qiu H, Wu H, Qian S. Efficacy and safety of decitabine in combination with G-CSF, low-dose cytarabine and aclarubicin in newly diagnosed elderly patients with acute myeloid leukemia. Oncotarget, 2015, 6: 6448-6458

7 Gatenby RA, Silva AS, Gillies RJ, Frieden BR. Adaptive therapy. Cancer Res, 2009, 69: 4894-4903

8 Benzekry S, Hahnfeldt P. Maximum tolerated dose versus metronomic scheduling in the treatment of metastatic cancers. J Theor Biol, 2013, 335: 235-244

9 Li SC, Lee KL, Luo J. Control dominating subclones for managing cancer progression and posttreatment recurrence by subclonal switchboard signal: implication for new therapies. Stem Cells Dev, 2012, 21: 503-506

10 Emadi A, Sadowska M, Carter-Cooper B, Bhatnagar V, van der Merwe I, Levis MJ, Sausville EA, Lapidus RG. Perturbation of cellular oxidative state induced by dichloroacetate and arsenic trioxide for treatment of acute myeloid leukemia. Leuk Res, 2015, 39: 719-729

Open Access This article is distributed under the terms of the Creative Commons Attribution License which permits any use, distribution, and reproduction in any medium, provided the original author(s) and source are credited. 